\title{
Pour en lire plus : Palo Alto à l'école
}

\section{Orane Bischoff}

\section{(2) OpenEdition \\ Journals}

Édition électronique

URL : http://journals.openedition.org/ere/570

DOI : $10.4000 /$ ere. 570

ISSN : 2561-2271

Éditeur

Centr'ERE

Référence électronique

Orane Bischoff, «Pour en lire plus : Palo Alto à l'école », Éducation relative à l'environnement [En ligne], Volume 12 | 2015, mis en ligne le 20 mai 2015, consulté le 24 septembre 2020. URL : http://

journals.openedition.org/ere/570; DOI : https://doi.org/10.4000/ere.570 


\title{
Pour en lire plus : Palo Alto à l'école
}

\author{
Orane Bischoff
}

\section{RÉFÉRENCE}

Michel Vidal et Teresa Garcia-Rivera (2013). Palo Alto à l'école. Montpellier : Editions SupAgro Florac

\section{Palo Alto à l'école}

\author{
par Michel Vidat et Terea Garci-Xivees
}

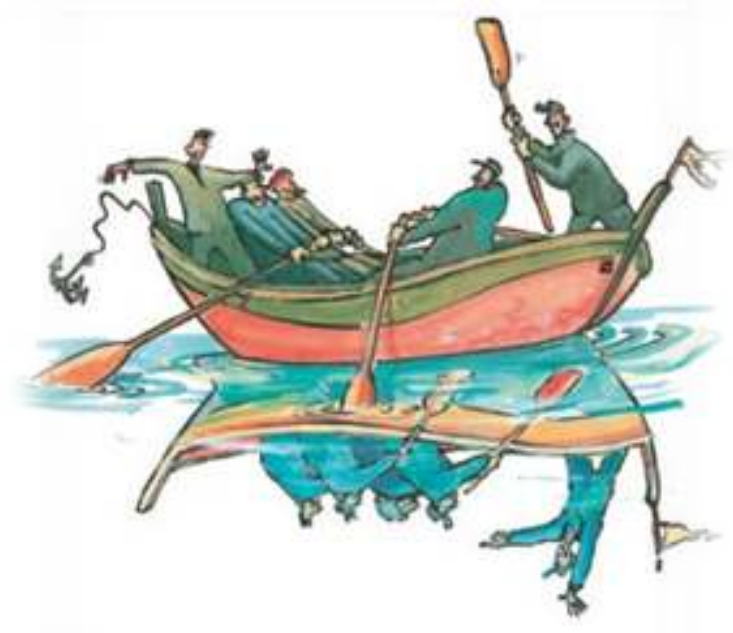

1 L'École de Palo Alto est un courant de pensée et de recherche ayant pris forme au début des années 1950 aux États Unis. Son approche s'inscrit initialement dans le champ de la communication interpersonnelle, la résolution des problèmes humains et la thérapie dite systémique. Elle interroge les principes interactionnels qui fondent les 
communications entre deux ou plusieurs personnes, interactions qui occupent tous les contextes de la vie.

2 L'ouvrage Palo Alto à l'école nous amène à explorer la relation éducateur/jeune dans le cadre scolaire sous un angle systémique ou cybernétique pour reprendre la terminologie de ce courant de pensée.

3 Les auteurs, Michel Vidal et Teresa Garcia-Rivera, partent du constat que le système scolaire est le lieu où se noue un certain nombre de problèmes et questionnements d'ordre psycho-social ayant un impact sur l'insertion professionnelle et sociale des jeunes, et que le personnel de l'équipe éducative se trouve à l'interface de ceux-ci. L'enjeu pourrait dès lors consister à outiller les membres de l'équipe éducative pour agir auprès des jeunes, et ce, sans être diplômés en psychologie. Dans un contexte où la relation est au cœur du système, mêlant divers niveaux d'interactions assez complexes avec des cadres formels souvent contraignants, Vidal et Garcia-Rivera pensent qu'une démarche s'inspirant de l'approche de Palo Alto offre une opportunité de renouveler cette relation d'accompagnement adulte/jeune. Sans pour autant remettre en cause d'autres pratiques, Michel Vidal et Teresa Garcia-Rivera proposent dans ce livre un éventail de pistes - qu'ils qualifient de "possibles" - à explorer qui s'inscrivent directement dans le courant de pensée de Palo Alto. En illustrant leurs propos par de nombreux témoignages d'éducateurs et de thérapeutes, ils présentent, en les alternant, les fondements théoriques de l'École de Palo Alto et les postures pratiques.

Le postulat envisagé par les auteurs est que la relation à l'autre est primordiale dans le bien-être de l'individu. D'ailleurs, les jeunes le confirment en faisant référence en premier lieu à leurs problèmes relationnels à l'école, au détriment de leurs difficultés d'apprentissage. C'est la relation aux autres et l'interaction avec son environnement qui est prioritairement mis en avant. Par conséquent, c'est bien sur la relation qu'il faut agir pour faire évoluer des situations de mal-être. Les auteurs suggèrent donc de porter un regard différemment et de s'interroger entre autres sur la manière dont les personnes interagissent entre elles et leur environnement. Dans l'école de pensée de Palo Alto, il s'agit donc de questionner le comment plutôt que le pourquoi qui se fonde sur des suppositions. Une telle démarche systémique suggère donc de saisir la trame des interactions en jeu pour en comprendre la dynamique ; elle vise à questionner la relation, permettant ainsi d'observer et d'aborder la situation différemment, voir autrement. Pour cela, il est important de s'inscrire dans une posture que prône l'école de Palo Alto, celle du paradoxe et de la double contrainte.

Dans cet ouvrage, les auteurs présentent les principes d'incertitude et de récursivité ainsi que différentes propriétés systémiques telles que : 1) un système est moins que la somme des propriétés de ses éléments, 2) Un système complexe possède une organisation « floue ", 3) Une partie du système ne peut pas contrôler l'ensemble, 4) Un système complexe est un système ouvert, 5) Un système complexe est à la fois robuste et sensible. Pour Michel Vidal et Teresa Garcia-Rivera, ces propriétés offrent des clés pour agir sur la dynamique relationnelle. Si un élément varie de façon significative, l'ensemble du système va changer.

6 Enfin, si le problème est dans la relation, la solution s'y trouve également. Cela veut dire que pour l'accompagnant, instaurer une relation "aidante» signifie ne pas imposer des normes ou des logiques extérieures, ce qui suppose de déployer une forme d'empathie et rentrer dans la logique de l'autre en évitant d'imposer sa propre représentation d'une solution "bonne" pour lui. La normativité est source de 
problème. Les auteurs citent Watzlawick : « le problème est la solution », ce qui conduit à questionner les représentations et les conceptions de l'individu dans un contexte donné. Dans cette école de pensée, le comment du geste de remédiation est plus important que l'introspection psychique.

7 Tout au long de cet ouvrage, Michel Vidal et Teresa Garcia guident l'éducateur vers la posture palo-altienne qui consiste souvent à prendre le problème tel qu'il se pose. Ils orientent l'accompagnant dans cette approche qui permet de sortir de la redondance et de la répétition pour envisager des solutions créatives, innovantes parfois inattendues, offrant ainsi au système relationnel une ouverture qui facilite une prise de décision plus émancipatrice et responsabilisante pour le jeune.

8 Les auteurs décodent plusieurs situations éducatives ou thérapeutiques en soulignant les gestes et postures innovantes mis en place par les éducateurs pour sortir des impasses relationnelles. Ils montrent le succès de cette approche par des résultats positifs sur les relations entre le jeune et le système scolaire.

9 L'ouvrage est passionnant à la fois par son décryptage de la pensée de Palo Alto et l'analyse de situations éducatives problématiques. Les pistes de remédiation présentées bouleversent nos postures quotidiennes dans la relation éducative.

Finalement, c'est un nouveau regard qui s'impose.

\section{AUTEURS}

\section{ORANE BISCHOFF}

Ingénieure d'étude pédagogique, Éducation, pédagogie et agro-environnement, SupAgro Florac 